\title{
Improving Tumor-Treating Fields with Skull Remodeling Surgery, Surgery Planning, and Treatment Evaluation with Finite Element Methods
}

\author{
Nikola Mikic and Anders R. Korshoej
}

\section{Introduction}

Tumor-treating fields (TTFields) are alternating fields $(200 \mathrm{kHz})$ used to treat glioblastoma (GBM), which is one of the deadliest cancer diseases of all. Glioblastoma is a type of malignant brain cancer, which causes significant neurological deterioration and reduced quality of life, and for which there is currently no curative treatment. TTFields were recently introduced as a novel treatment modality in addition to surgery, radiation therapy, and chemotherapy. The fields are induced noninvasively using two pairs of electrode arrays placed on the scalp. Due to low electrical conductivity, significant currents are shielded from the intracranial space, potentially compromising treatment efficacy. Recently, skull remodeling surgery (SR-surgery) was proposed to address this issue. SR-surgery comprises the formation of skull defects or thinning of the skull over the tumor to redirect currents toward the pathology and focally enhance the field intensity. Safety and feasibility of this concept were validated in a clinical phase 1 trial (OptimalTTF-1), which also indicated promising survival benefits. This chapter describes the FE methods used in the OptimalTTF-1 trial to plan SR-surgery and assess treatment efficacy. We will not present detailed modeling results from the trial but rather general concepts of

\footnotetext{
N. Mikic

Department of Neurosurgery, Aarhus University Hospital, Aarhus, Denmark

Department of Clinical Medicine, Aarhus University, Aarhus, Denmark

Department of Neurosurgery, Aalborg University Hospital, Aalborg, Denmark
}
A. R. Korshoej ( $\square)$
Department of Neurosurgery, Aarhus University Hospital, Aarhus, Denmark
Department of Clinical Medicine, Aarhus University, Aarhus, Denmark
e-mail: andekors@rm.dk 
model development and field calculations. Readers are kindly referred to Wenger et al. [1] for a more general overview of the clinical implications and applications of TTFields modeling.

\section{Glioblastoma}

GBM is the most common and one of the most aggressive primary malignant tumors in the central nervous system [2]. GBM is a WHO grade IV glial tumor characterized by invasive growth and significant anaplasia. The age-standardized incidence rate of GBM in Denmark is 6.3/100,000 person-years for males and 3.9/100,000 personyears for females with a median age of 66 years and a median overall survival of 11.2 months [3], which corresponds well with survival estimates from other Western countries [4]. Today standard therapy consists of maximal surgical resection followed by radiotherapy with concomitant and adjuvant temozolomide chemotherapy [5].

\section{Tumor Treating Fields}

In the search for new treatment options for GBM, TTFields have recently been introduced as a fourth and supplementary treatment modality applied in parallel with adjuvant temozolomide. TTFields are alternating electric fields of low intensity $(100-500 \mathrm{~V} / \mathrm{m})$ and intermediate frequency $(200 \mathrm{kHz})$ that are transmitted through the head and brain between electrodes placed noninvasively in an individualized pattern on the patient's scalp (Fig. 1). The electric fields affect dividing cells in particular and hereby primarily cancer cells. The therapeutic effect of TTFields is explained by two physical principles, dielectrophoresis and dipole alignment. In combination, the two principles affect the normal movement of charged and polarizable structures, including septin and tubulin, which is highly responsible for successful mitosis. Thus the disruption of these mechanisms leads to cell death [1]. In patients with newly diagnosed GBM, TTField therapy in combination with chemotherapy has been proved to have a significant effect on median overall survival (OS) and median progression-free survival (PFS) compared to chemotherapy alone [6]. A recent meta-analysis of studies on TTField treatment of GBM patients further concludes that TTFields are an efficient and safe treatment modality [7]. The positive effects of TTFields, recently, led to the introduction of TTFields as a category 1 recommendation of TTFields for a selected population of patients with newly diagnosed GBM by the National Comprehensive Cancer Network in the USA [8]. 

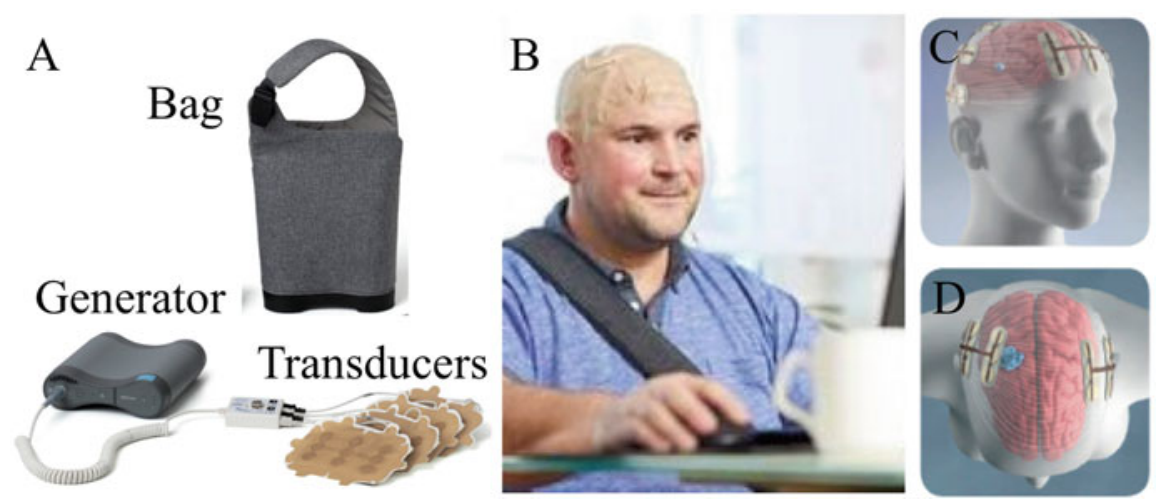

Fig. 1 TTField therapy. Two pairs of electrode arrays are connected to a TTField generator carried by the patient in a bag (a). The arrays are placed on the patient's head (b). Each array pair induces alternating TTFields in sequence (c) using a 50:50 duty cycle. (The patient photograph is published with permission from the patient (Courtesy of Novocure). The figure is adapted from Korshoej et al. [10])

In regard to the practical use of TTFields, patients are recommended to wear the active device as much as possible - designated as the level of compliance. A compliance threshold above 50\% correlates positively with improved outcome, but maximal effect on survival rates is attained with a compliance of $>90 \%$ [9], and therefore continuous treatment is recommended whenever possible.

\section{TTFields Dosimetry}

In recent years, finite element $(\mathrm{FE})$ methods have been used to estimate the distribution of TTFields intensity in the patient's head and tumor with the objective of improving technology design and treatment implementation. The rationale behind this approach is that high field intensities correlate positively with longer overall survival [11] and increased tumor kill rate in vitro $[12,13]$, so field estimation can be considered an approach to TTField dosimetry with potential applications for individual treatment planning as well as identification of expected responders to therapy and prediction of the expected treatment prognosis and topographical patterns of recurrence in the brain. Although previous studies have established that field intensity is a highly relevant surrogate dose parameter, it is well-known that other factors such as field frequency, treatment duration, and spatial correlation also affect the efficacy of TTFields [14-16]. Ongoing work is being conducted to refine the dosimetry methods and establish a golden standard with a strong correlation to clinical outcome. 


\section{Skull Remodeling Surgery and the Utility of FE Modeling}

As an example of FE modeling utility, we recently demonstrated that the high resistivity of the skull causes significant amounts of currents to be shielded from the intracranial regions of interest, which may compromise treatment efficacy. To overcome the obstacle, we proposed a surgical skull remodeling procedure (SR-surgery) aiming to introduce localized skull defects (with reduced skull resistivity) and thereby redirect the tumor inhibiting currents toward the underlying regions of interest (Fig. 2) [17]. SR-surgery encompasses thinning of the skull or formation of burr holes or larger skull defects (craniectomies) over the tumor region, which causes the intensity of the field (i.e., treatment dose) to increase in these regions (Fig. 3) and further reduces the amount of wasted electrical energy deposited in the skin (Fig. 2b).

In search of a feasible approach for clinical implementation, we previously explored a number of different configurations of craniectomy and found that the field intensity in the underlying tumor increases with craniectomy diameter, until the skull defect is approximately the same size as the underlying region of interest. When the defect area exceeds the size of the underlying pathology, it causes currents to be shunted around and pass the intended target and therefore does not contribute to further dose enhancement in the desired area (Fig. 4). In addition, we found that it was more effective to use multiple smaller burr holes distributed over the region of interest, rather than a single craniectomy. With this approach it was possible to achieve higher field enhancement per skull defect area, which made the approach favorable from a clinical safety perspective.

Recently, we demonstrated the safety and feasibility of the SR-surgery concept in a clinical phase 1 trial (OptimalTTF-1, clinicaltrials.gov ID: NCT02893137). We found that SR-surgery combined with TTFields was not associated with serious adverse events related to the intervention, and adverse events observed could be attributed to medical therapy or TTField treatment alone. In addition, the trial further indicated a promising treatment efficacy with prolonged overall survival and progression-free survival compared to historical data from comparable patient cohorts [18].

\section{The Aim and Motivation of Field Modeling in SR-Surgery Planning and Evaluation}

In the OptimalTTF-1 trial, we used field modeling for a number of purposes. The most important motivation was the need for a method to ensure that enrolled patients would gain an expected benefit from the participation in the trial. Since all enrolled patients underwent SR-surgery, and thereby had to accept the potential risks of the surgery itself in addition to the risks associated with reduced skull protection in the operated region, we required the expected dose enhancement to be considerable for 


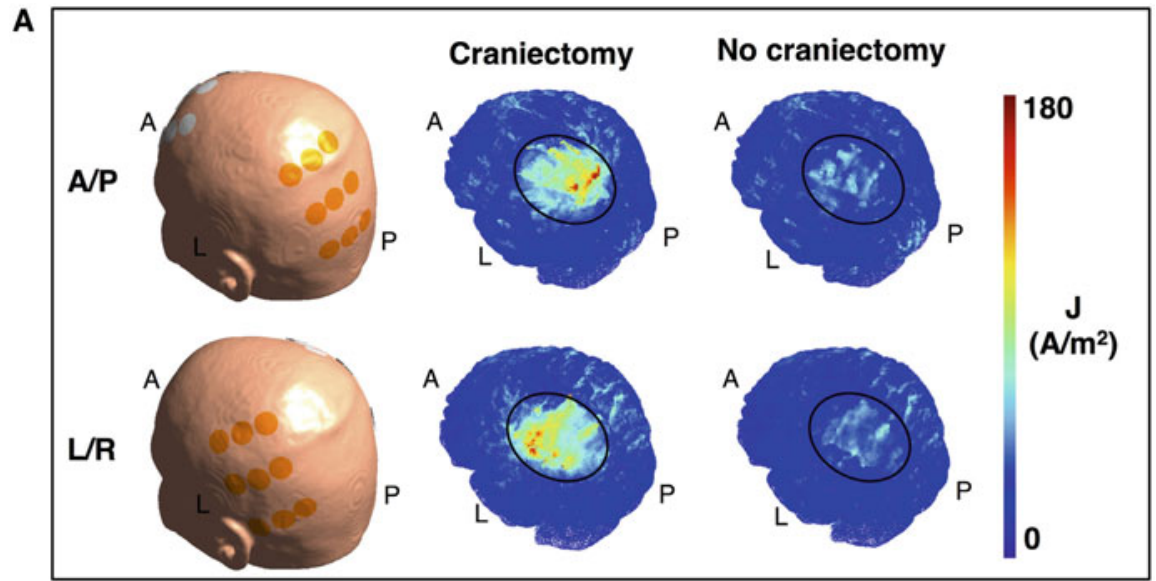

B

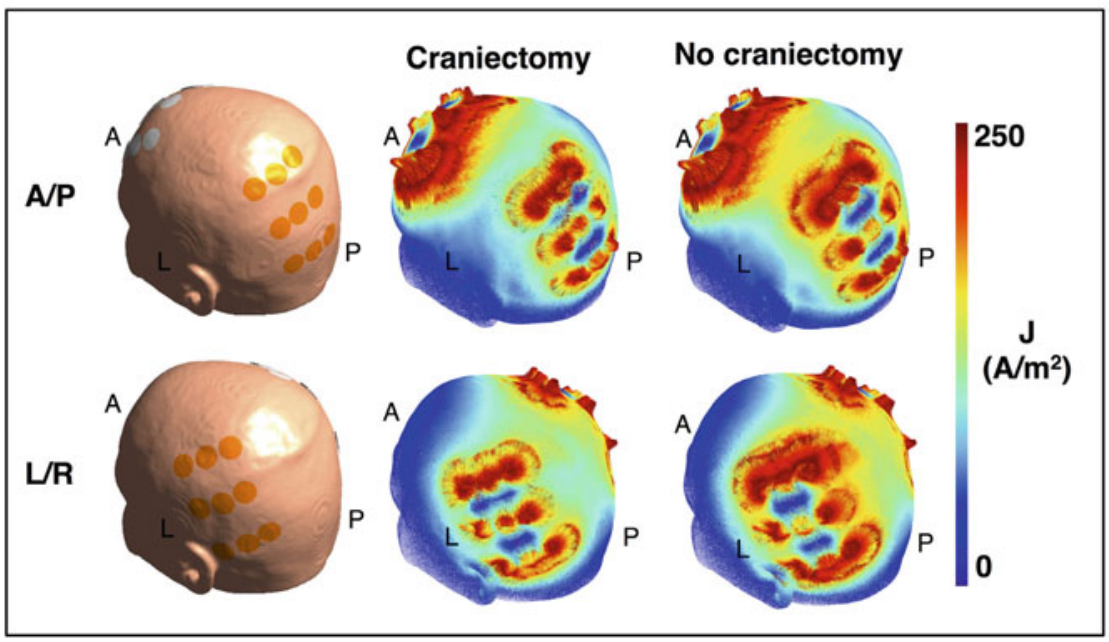

Fig. 2 Effect of craniectomy on the field and current distribution in a human head model. (Reproduced from Korshoej et al. [17]). (a) Surface representations of a patient's head with the left/right (L/R) and anterior/posterior (A/P) array pairs positioned on the scalp. The middle panel shows the current density distribution on the brain surface induced by the corresponding array configurations in the presence of a craniectomy (encircled) above the tumor region. Compared to a situation with no craniectomy (right-most panels), it is clear that craniectomy causes a significant amount of current to flow through the craniectomy and toward the underlying brain region. (b) This panel shows results similar to (a), but with the current distributions shown for the skin and electrode surfaces, respectively. The craniectomy redistributes how the impressed currents flow through the electrodes, and more importantly it causes a lower amount of current to flow through the skin between the electrodes and rather redirects the current toward the brain region underneath the hole in the skull

ethical reasons. Therefore, we set the threshold to an average expected field enhancement of $>25 \%$ in the region of pathology, i.e., the remnant tumor or the peritumoral border zone. This was assessed using a reasonably quick and flexible modeling 
A

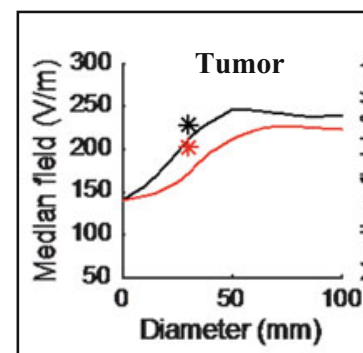

No resection

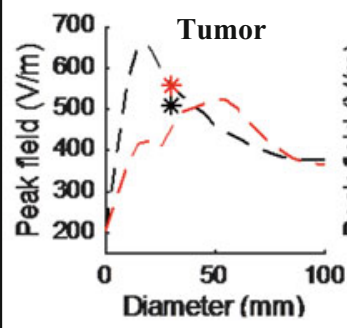

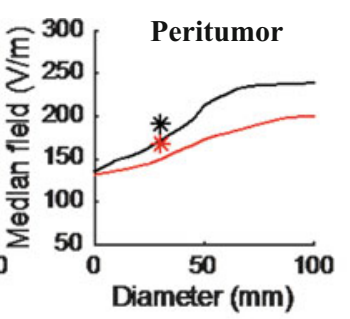

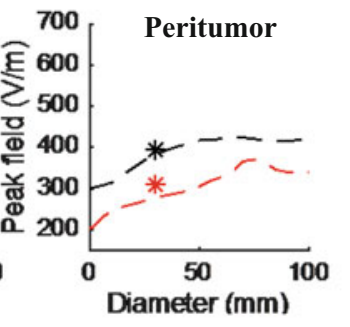

\section{B Resection}

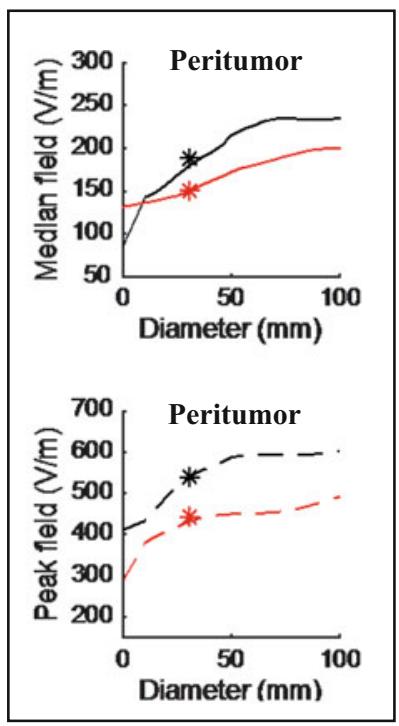

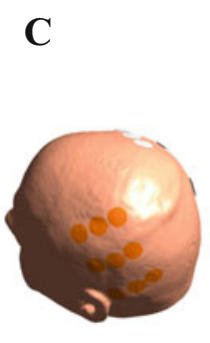
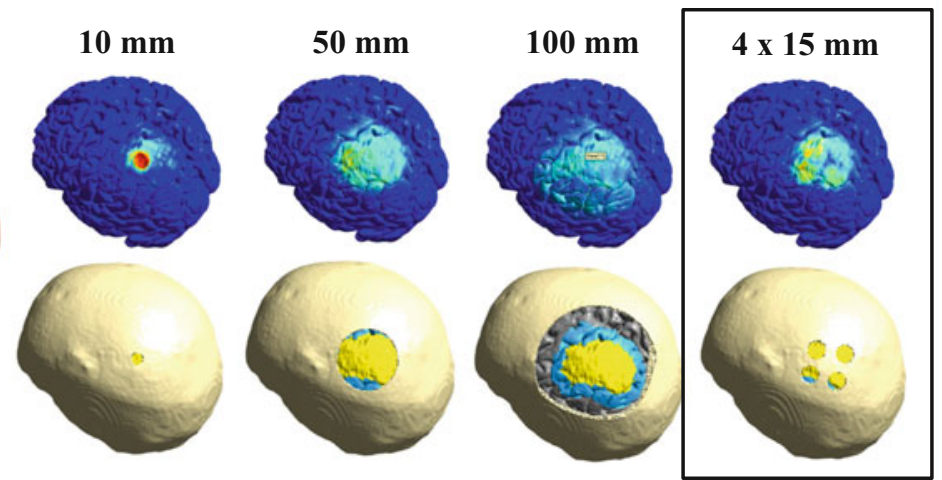

Fig. 3 Effect of different craniectomy configurations. (a) This panel shows the peak field and median field values in the tumor region and peritumoral region $(2 \mathrm{~cm}$ around the tumor), when no resection is performed, for different sizes of circular craniectomies. The red line represents the anterior/posterior array pair, and the black line represents the left/right pair. The enhancing effect of the craniectomies tends to plateau around a diameter of 5-7 cm, equivalent to the size of the underlying tumor. The asterisks represent the equivalent values for a configuration with four $15 \mathrm{~mm}$ burr holes distributed over the tumor. This configuration is equally effective as a 5 -cm-diameter craniectomy. (b) This panel shows results similar to panel a, but following resection. The same conclusions apply although the plateau tendency is less pronounced in the given case. (c) This panel shows examples of the investigated craniectomy configurations with the underlying brain, tumor, and peritumoral region and field intensity in the brain surface. (The figure is reproduced from Korshoej et al. [17]) 


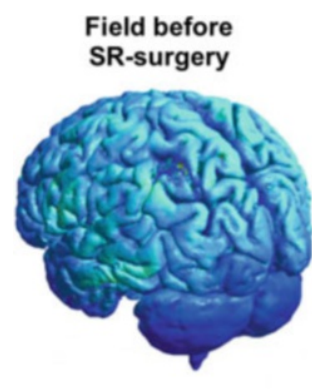

0

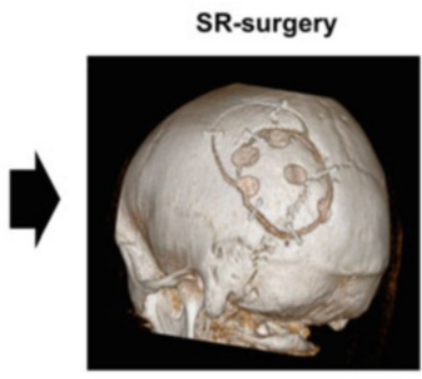

TTFields intensity $(\mathrm{V} / \mathrm{m})$

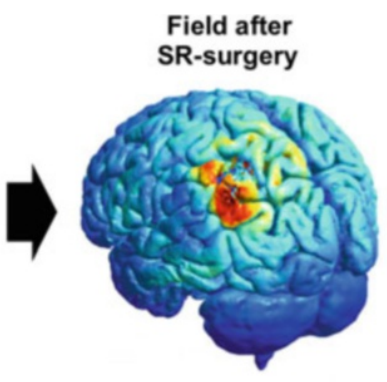

450

Fig. 4 The effect of skull remodeling in a single-trial case. Surface representation of the field intensity distribution in the brain and resection cavity from a patient in the OptimalTTF-1 trial. Furthermore, the middle panel shows the SR-surgery configuration applied for the given patient, and the right panel shows the field distribution after the craniectomy. SR-surgery caused the field in the peritumoral region around the resection cavity to be enhanced by approximately $50 \%$ in the given case. (This figure is adapted from Korshoej et al. [10])

approach, in which a tumor mimicking the actual patient case were introduced virtually in a preexisting computational head model based on MRI data from a healthy individual (see below). The reason for adopting the approach was that we needed a technique for quick evaluation and exploration of SR-surgery benefit in various configurations. In Denmark, there is a legal requirement to initialize treatment of cancer patients (i.e., operate in this case) within 2 weeks of suspected tumor diagnosis or establishment of disease progression. Therefore, it was not possible to construct detailed and personalized head models for each enrolled patient prior to surgery, as this procedure is very time-consuming. Instead, we used the flexible approach, with which model creation and surgery planning could be completed within approximately 2 days. The computations were initiated immediately upon patient enrollment. We used the model to explore different SR-surgery configurations and identify the optimal configuration with the highest field gain possible for each patient. This configuration was then used to guide the surgery. As a predefined rule, the total skull defect area had to be $<30 \mathrm{~cm}^{2}$.

In addition to validating treatment benefit, an important motivation was to be able to correlate topographical patterns of disease recurrence on MRI with detailed individual assessments of the TTField distribution in treated patients. This work is exploratory in nature and requires accurate computational models based on MRI data from individual patients. Moreover, these more accurate models would serve to validate the estimates obtained in the preliminary preoperative simulations. This work is still ongoing and beyond the scope of the present paper, but the concept illustrates how FE modeling may be used to address and explore many clinically relevant aspects of TTField therapy. The following sections will focus on describing the basic framework of the quick and flexible modeling technique that was used for the assessment of treatment benefit upon patient enrollment. 


\section{Physical Basis of the Field Calculations}

Before we continue to discuss the construction of the head models, we will briefly present the physical framework assumed for the calculations. Given the dielectric properties of biological tissues, the low to intermediate frequency of TTFields $(200 \mathrm{kHz}$ ), and the small width of the head (approximately $20 \mathrm{~cm}$ ) [19], we can assume TTFields to behave in a quasi-stationary fashion. Therefore, the electric potential $\varphi$ can be approximated with Laplace's equation

$$
\nabla \cdot(\sigma \nabla \varphi)=0
$$

where $\nabla \cdot$ is the divergence operator and $\sigma$ is the real-valued conductivity [20]. In our calculations, we used the FE approach to obtain an approximate numerical solution to Laplace's equation of the electrostatic potential. The field distribution was then initially derived by taking the gradient of the potential distribution and the current density subsequently from Ohm's law and using the derived field and the scalar conductivity assigned to the element. All distributions were calculated separately for each of the electrode pairs, as they are activated sequentially in the real treatment scenario. In addition, calculations were performed both before and after introducing a virtually planned SR-surgery procedure into the model. This allowed us to calculate the absolute and relative changes in the average field intensity in the respective regions of interest, including the tumor and peritumoral border zone, and thereby to quantify the expected field enhancement caused by the intervention.

\section{Creating the Head Models}

The head models used for computations were constructed from the dataset "almi5," which was created using SimNIBS [21] and which is available from simnibs.org. The model was initially composed of five volumes, namely, skin, skull, cerebrospinal fluid (CSF), gray matter (GM), and white matter (WM). To incorporate the tumor, necrotic regions, and resection cavities, we post-processed the surface mesh STL files of the model for every patient. The post-processing was based on morphological measurements of the pathology regions on preoperative MRI images of the patient, including gadolinium-enhanced T1 sequences. The tumor was incorporated into the GM volume, the necrotic region into the tumor interior, and the resection cavity into the CSF volume. The edited surface meshes were "cleaned" for self-intersections and triangle degenerations using MeshFix. Subsequently, all volumes encapsulated by neighboring surfaces were tessellated with Gmsh (gmsh. info) to construct a tetrahedral computational mesh. The skull defects, i.e., virtual SR-surgeries, were initially outlined in MeshMixer by producing closed (often spherical or cylindrical) compact surface files traversing the exterior and interior boundaries of the skull in a desired geometrical configuration and location. These 

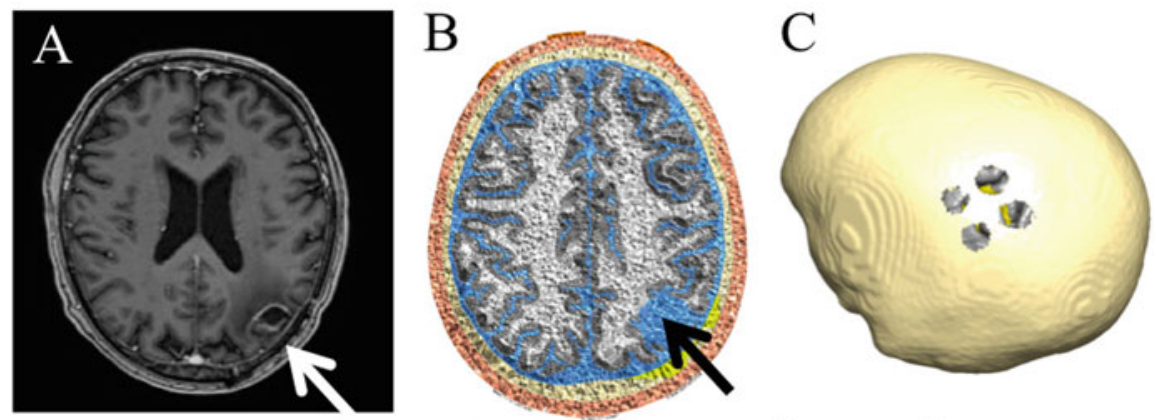

\section{Tumor/Resection cavity Resection cavity}
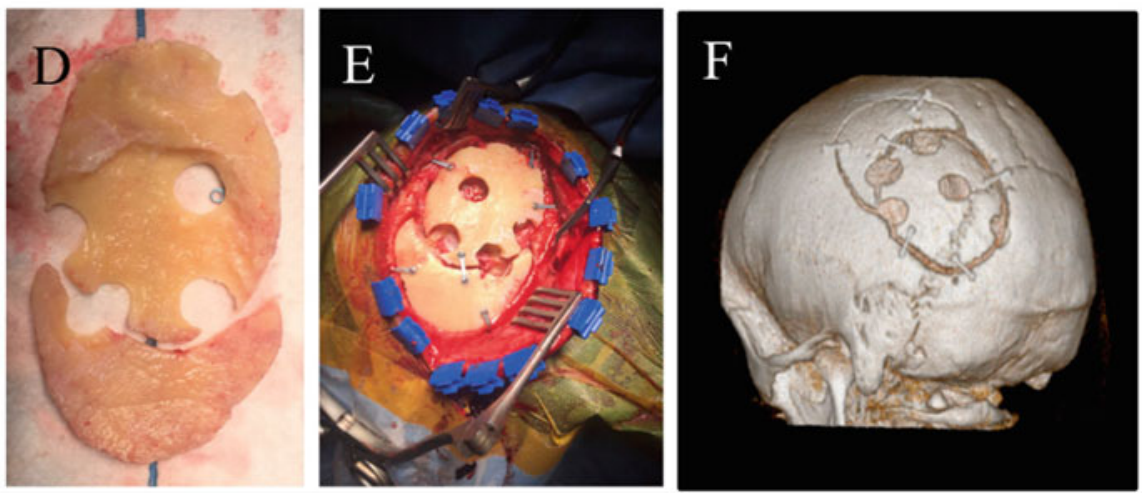

Fig. 5 SR-surgery planning for the patient shown in Fig. 4. (a) Contrast-enhanced T1 MRI showing the tumor/resection. (b) Patient head model showing approximation of intended resection cavity. (c) Outline of the SR-surgery plan. (d and e) Images of the remodeled bone plate. Four burr holes ( $15 \mathrm{~mm}$ diameter) were created and the interior plate thinned out in a 5 -cm-diameter area over the tumor. (f) CT scan of the SR-surgery result. (The figure and legend is reproduced from Korshoej et al. [10])

volumes were then used to define binary volume masks used to select the elements to be contained in the surgical skull defects. These elements were then assigned a uniform isotropic conductivity equal to the skin, based on the assumption that the removed skull tissue would be replaced with a better-conducting skin tissue. The holes in the skull were typically placed directly above the tumor and resection border. A number of configurations were then tested in a trial-and-error fashion, and the model selected for SR-surgery was then visualized using Gmsh and used as a guiding framework for surgery in combination with neuronavigation technologies (Fig. 5). 


\section{Placement of TTField Transducer Arrays}

The $3 \times 3$ TTField electrode arrays were positioned to maximize TTField intensity for each patient and portray the clinical treatment scenario planned for the individual patient. In a normal clinical setting, the array layout is determined using the NovoTAL ${ }^{\circledR}$ software (Novocure ${ }^{\mathrm{TM}}$ ). NovoTAL ${ }^{\circledR}$ uses individual measurements of the head size and tumor size/position to design a layout for each treated individual, which maximizes the field intensity in the tumor. However, the alteration and redistribution of the current density and electric field caused by SR-surgery arguably invalidate this approach, and we therefore planned the array layouts using the guiding principles of optimized and individualized array placement outlined in Korshoej et al. [22, 23] as well as generalized principles determining the distribution of TTFields [24, 25]. Basically, the arrays were placed so that a row of edge transducers from one array in each pair overlaid the tumor (Fig. 6) and the remodeled region of the skull, while the other array in the same pair was placed on opposite side of the skull, ensuring that currents would flow through the holes in the skull and toward the opposite side of the head and thereby induce high fields in the tumor. This approach is based on the observation that stronger fields are induced in tissues underlying the periphery of the electrode arrays ("edge effect"). Hence, it is not desirable to have the skull holes located under the central parts of the array or in a far distance from the array, as this would reduce the amount of current likely to pass through the holes. The virtual placement of electrodes was performed using the SimNIBS GUI and a custom Matlab script (Mathworks, Inc.). For further details, see [23].

\section{Boundary Conditions and Tissue Conductivities}

Computations were conducted using the Dirichlet boundary conditions defined by the anatomical boundaries of the head and fixed electrical potentials at the top of the array transducers. Particularly, the potential was set to $1 \mathrm{~V}$ in the transducers of one array in a pair, while the potential in the electrodes of the other array were set to $-1 \mathrm{~V}$. Numerical approximation was obtained using a conjugate gradient solver with a defined tolerance of 1 E-9. All potentials, fields, and current densities were then rescaled to obtain a total current of 1.8A through the arrays equivalent to the amount of current delivered by the Optune ${ }^{\mathrm{TM}}$ device. This allowed us to model the actual scenario that all electrodes in an array were connected to the same electrical source. In all calculations, a uniform isotropic scalar conductivity value $\sigma$ was assigned to all nodes in a volume based on previous measurements from in vitro and in vivo studies (skin $0.25 \mathrm{~S} / \mathrm{m}$, bone $0.010 \mathrm{~S} / \mathrm{m}, \mathrm{CSF} 1.654 \mathrm{~S} / \mathrm{m}$, tumor $0.24 \mathrm{~S} / \mathrm{m}$, and necrosis $1.00 \mathrm{~S} / \mathrm{m}$ [23]). All transducers were modeled with an underlying layer of conductive gel with $0.5 \mathrm{~mm}$ thickness and $1.0 \mathrm{~S} / \mathrm{m}$ conductivity. 
A
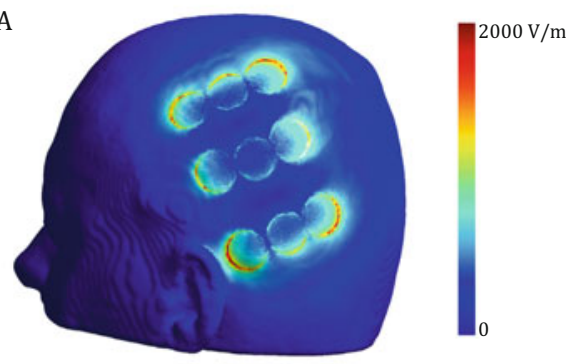

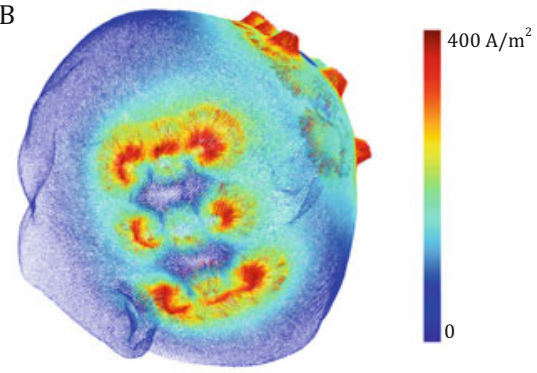

c

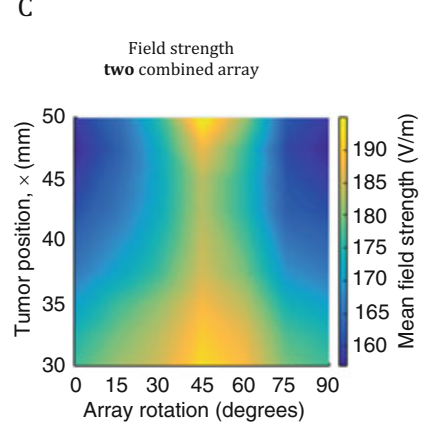

D

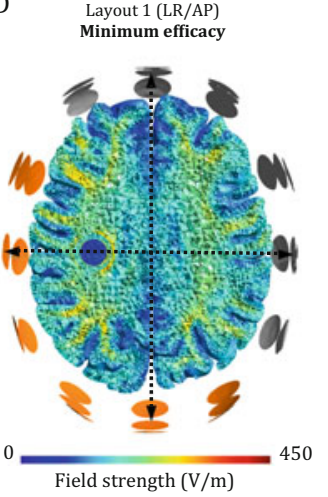

E Layout 2 (oblique) Maximum efficacy

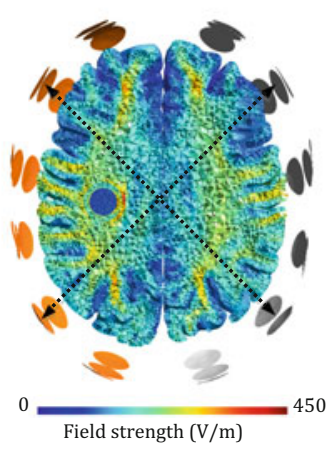

Fig. 6 The edge effect and principles of electrode array positioning. The panels a and $\mathbf{b}$, respectively, show the skin surface representations of the current density (a) and field intensity (b) induced by the left/right array pair of a participant in the trial. Both panels illustrate that the stronger fields and currents are present near the periphery of the array. Panels $\mathbf{c}-\mathbf{e}$ illustrate the underlying principle adopted when placing the arrays on the head of the patient. Panel $\mathrm{c}$ shows the mean field intensity in a virtually introduced 2-cm-diameter tumor with a 1.4-cm-diameter central necrotic core for different tumor positions and array positions. Specifically, we tested how the field was affect by 15-degree stepwise rotations of an orthogonal configuration of two array pairs in the same horizontal plane (b and $\mathbf{c}$ ). This rotation was conducted around a central craniocaudal axis. Eleven tumors were investigated for all rotations. Particularly, the tumors were translated along an axis in the coronal plane from deep positions ( $30 \mathrm{~mm}$ from the median plane) to superficial positions (50 $\mathrm{mm}$ from the median plane). The tumors were located in the plane of the central transducers of the arrays. For all tumors, the maximum average field intensity was achieved when the array pairs were oriented both at 45 degrees to the sagittal plane, i.e., obliquely (panel e). The default layout (i.e., anterior/posterior and left/right, panel d) were the least efficient for these tumors. These results are further elaborated in Korshoej et al. [23] from which this figure has been adapted. The conclusions of these investigations are that arrays should be placed such that the edge of one array from each pair is placed in close vicinity to the tumor (and the introduced skull defects) and the other array in the same pair on the opposite side of the head. This approach was also adopted when positioning the arrays in the OptimalTTF-1 trial. (Panels $\mathbf{c}-\mathbf{e}$ of this figure are reproduced from Korshoej et al. [23]) 


\section{SR-Surgery in the OptimalTTF-1 Trial}

In the OptimalTTF-1 trial, a total of 15 subjects were enrolled. The tumors were located in the temporal $(N=5)$, parietal $(N=2)$, frontal $(N=2)$, occipital $(N=1)$, frontoparietal $(N=3)$, and parietooccipital $(N=2)$ regions, and field enhancement $>25 \%$ could be obtained for all patients (median 37\%, range 25-67\%). The applied skull defects had a mean area of $10.5 \mathrm{~cm}^{2}$ (range $7-24 \mathrm{~cm}^{2}$ ), and the mean absolute field values in the region of interest were in the range 100-200 V/m. Ten patients had 4-6 burr holes (15-18 mm diameter), and two had total craniectomies (elliptic with semiaxis diameters of approximately $60 \times 50 \mathrm{~mm}$ and $85 \times 65 \mathrm{~mm}$, respectively). One had five $15 \mathrm{~mm}$ burr holes and one $25 \mathrm{~mm}$ mini-craniectomy, while the remaining two patients had seven and eight $20 \mathrm{~mm}$ burr holes, respectively. Figure 7 shows examples of two different configurations of SR-surgery, while a third example is given in Fig. 5f. The remodeled regions were placed above the resection cavity/border and residual tumor. Skull thinning was performed if possible and if the resection cavity extended to regions where the overlying skull had an estimated thickness above $3 \mathrm{~mm}$. Skull thinning in areas below this limit was considered less significant because the relative gain in conductivity would be too small in these cases. For patients with temporal tumors, the squamous area of the temporal bone was therefore only perforated by burr holes, and bone bridges were left to support the overlying temporal muscle and maintain cosmetic integrity. All surgeries were conducted by trained neurosurgeons. The operation was technically feasible, easy

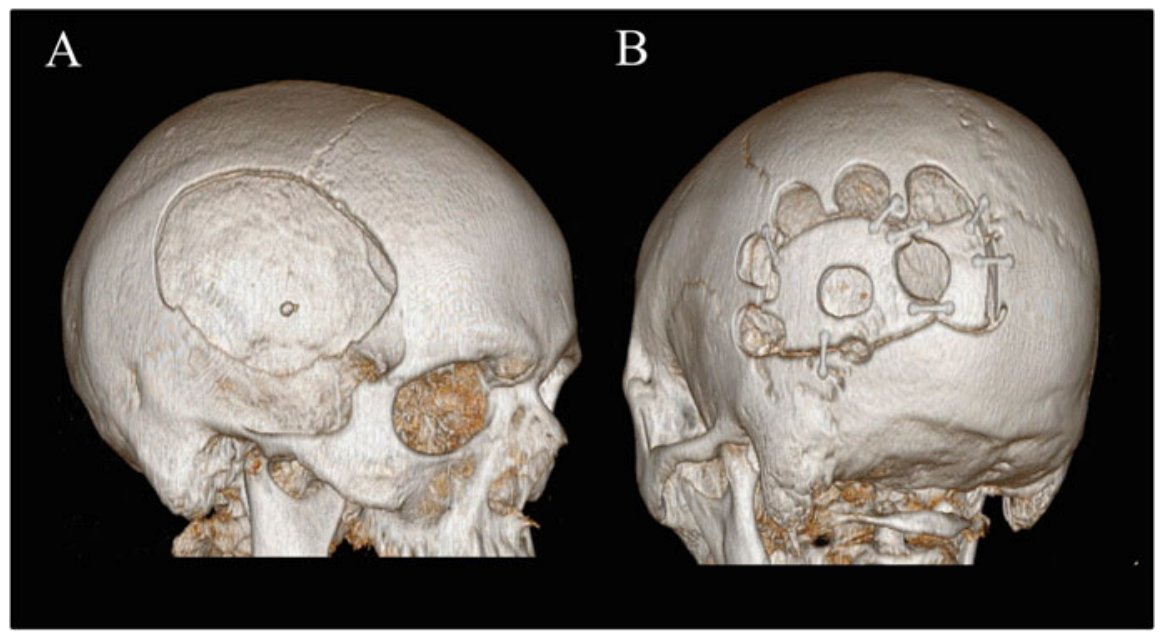

Fig. 7 CT reconstructions of two additional examples of SR-surgery configurations. (a) Total craniectomy $(85 \times 65 \mathrm{~mm}$, elliptic) above the tumor region. This was equivalent to a standard craniotomy bone flap created during resection surgery. (b) Seven burr holes (18 $\mathrm{mm}$ diameter) distributed above the resection cavity and its surrounding borders, tumor region before and after SR-surgery. (This figure is reproduced from Korshoej et al. [10]) 
to perform, and added less than 15 min of additional surgery time. Overall survival was 15.0 months, CI95\% $=[9.6 ; 16.2]$, and the overall survival rate at 1 year was $64 \%, \mathrm{CI} 95 \%=[35 ; 85]$, which is promising compared to historical data.

\section{Conclusion}

In this chapter, we have introduced the general concept of TTFields and well as background information on the main indication of this treatment, i.e., glioblastoma. We have illustrated the technical framework and rationale for implementation FE modeling dosimetry as a method to plan and evaluate skull remodeling surgery in combination with tumor-treating field therapy of GBM. We have illustrated how SR-surgery can be used to increase the TTField dose in GBM tumors and the techniques used to quantify this enhancement. The presented framework was adopted in a phase 1 clinical trial to validate expected efficacy for patients enrolled in the trial and further to calculate the field enhancement achieved for each patient. The trial, which is concluded at this time, showed that the SR-surgery approach was safe, feasible, and potentially improved survival in patients with first recurrence of GBM [18]. Two different modeling approaches were adopted, namely, a fast but less accurate approach, in which a representative tumor or resection cavity was introduced virtually in a computational model based on a healthy individual and one based on the individual patients MRI data, which was more accurate but also too time-consuming to be used for quick preoperative calculations. Here we have mainly focused on describing the principles and workflow of the simplified framework. Although we considered this approach sufficient for the given purpose, future work is needed to improve the FE pipeline for better time-efficiency and preparation of patient-specific models as exemplified in [17]. Such models would both improve anatomical accuracy and also allow for individualized anisotropic conductivity estimation giving a more accurate and realistic basis for the calculations. In the OptimalTTF-1 trial, we conducted the necessary MRI scans for individualized modeling preoperatively, postoperatively, and at disease recurrence for most patients. Based on this data, we aim to conduct individualized and refined post hoc simulations to accurately reproduce the actual skull remodeling configurations including skull thinning and thereby provide more accurate estimates of the beneficial effect of SR-surgery. This will be highly valuable when exploring the doseresponse relationship and effects of craniectomy enhancement of TTFields in further detail. Furthermore, efforts are being made to streamline and automate the simulation pipeline to enable quick and accurate dose estimation and treatment planning before SR-surgery. Such procedures would ideally also use automated optimization procedures as opposed to the current exploratory approach to ensure maximal dose enhancement. Finally, we are finalizing the analysis of the OptimalTTF-1 trial, which will shed important light to the clinical significance of the concept. A future clinical phase 2 trial is being planned to test treatment efficacy. 


\section{References}

1. Wenger, C., Miranda, P., Salvador, R., Thielscher, A., Bomzon, Z., Giladi, M., et al. (2018). A review on Tumor Treating Fields (TTFields): Clinical implications inferred from computational modeling. IEEE Reviews in Biomedical Engineering, 11, 195.

2. Omuro, A., \& DeAngelis, L. M. (2013). Glioblastoma and other malignant gliomas: A clinical review. JAMA, 310(17), 1842-1850.

3. Hansen, S., Rasmussen, B. K., Laursen, R. J., Kosteljanetz, M., Schultz, H., Nørgård, B. M., et al. (2018). Treatment and survival of glioblastoma patients in Denmark: The Danish neurooncology registry 2009-2014. Journal of Neuro-Oncology, 139(2), 479-489.

4. Ostrom, Q. T., Gittleman, H., Liao, P., Vecchione-Koval, T., Wolinsky, Y., Kruchko, C., et al. (2017). CBTRUS statistical report: Primary brain and other central nervous system tumors diagnosed in the United States in 2010-2014. Neuro-Oncology, 19(Suppl 5), v1-v88.

5. Weller, M., van den Bent, M., Hopkins, K., Tonn, J. C., Stupp, R., Falini, A., et al. (2014). EANO guideline for the diagnosis and treatment of anaplastic gliomas and glioblastoma. The Lancet Oncology, 15(9), e395-e403.

6. Stupp, R., Taillibert, S., Kanner, A., Read, W., Steinberg, D. M., Lhermitte, B., et al. (2017). Effect of tumor-treating fields plus maintenance temozolomide vs maintenance temozolomide alone on survival in patients with glioblastoma: A randomized clinical trial. JAMA, 318(23), 2306-2316.

7. Magouliotis, D. E., Asprodini, E. K., Svokos, K. A., Tasiopoulou, V. S., Svokos, A. A., \& Toms, S. A. (2018). Tumor-treating fields as a fourth treating modality for glioblastoma: A meta-analysis. Acta Neurochirurgica, 160, 1-8.

8. National Comprehensive Cancer Network. (2017). NCCN guidelines version 1.2017. Sub-Committees Central Nervous System Cancers.

9. Toms, S., Kim, C., Nicholas, G., \& Ram, Z. (2018). Increased compliance with tumor treating fields therapy is prognostic for improved survival in the treatment of glioblastoma: A subgroup analysis of the EF-14 phase III trial. Journal of Neuro-Oncology, 141, 1-7.

10. Korshoej, A. R., Mikic, N., Hansen, F. L., Thielscher, A., Saturnino, G. B., \& Bomzon, Z. (2019). Enhancing tumor treating fields therapy with skull-remodeling surgery. The role of finite element methods in surgery planning. 201941 st annual international conference of the IEEE Engineering in Medicine and Biology Society (EMBC) (pp. 6995-6997). IEEE.

11. Ballo, M. T., Urman, N., Lavy-Shahaf, G., Grewal, J., Bomzon, Z., \& Toms, S. (2019). Correlation of tumor treating fields dosimetry to survival outcomes in newly diagnosed glioblastoma: A large-scale numerical simulation-based analysis of data from the phase $3 \mathrm{EF}-14$ randomized trial. International Journal of Radiation Oncology, Biology, Physics, 104(5), $1106-1113$.

12. Kirson, E. D., Dbaly, V., Tovarys, F., Vymazal, J., Soustiel, J. F., Itzhaki, A., et al. (2007 Jun 12). Alternating electric fields arrest cell proliferation in animal tumor models and human brain tumors. Proceedings of the National Academy of Sciences of the United States of America, 104 (24), 10152-10157.

13. Kirson, E. D., Gurvich, Z., Schneiderman, R., Dekel, E., Itzhaki, A., Wasserman, Y., et al. (2004). Disruption of cancer cell replication by alternating electric fields. Cancer Research, 64 (9), 3288-3295.

14. Korshoej, A. R., \& Thielscher, A. (2018). Estimating the intensity and anisotropy of tumor treating fields using singular value decomposition. Towards a more comprehensive estimation of anti-tumor efficacy. 2018 40th Annual international conference of the IEEE Engineering in Medicine and Biology Society (EMBC). IEEE.

15. Korshoej, A. R., Sørensen, J. C. H., Von Oettingen, G., Poulsen, F. R., \& Thielscher, A. (2019). Optimization of tumor treating fields using singular value decomposition and minimization of field anisotropy. Physics in Medicine \& Biology, 64(4), 04NT03.

16. Korshoej, A. R. (2019). Estimation of TTFields intensity and anisotropy with singular value decomposition: A new and comprehensive method for dosimetry of TTFields. In Brain and 
human body modeling: Computational human modeling at EMBC 2018 (pp. 173-193). Cham: Springer.

17. Korshoej, A. R., Saturnino, G. B., Rasmussen, L. K., von Oettingen, G., Sørensen, J. C. H., \& Thielscher, A. (2016). Enhancing predicted efficacy of tumor treating fields therapy of glioblastoma using targeted surgical craniectomy: A computer modeling study. PLoS One, 11(10), e0164051.

18. Korshoej, A., Lukacova, S., Sørensen, J. C., Hansen, F. L., Mikic, N., Thielscher, A., et al. (2018). ACTR-43. Open-label phase 1 clinical trial testing personalized and targeted skull remodeling surgery to maximize ttfields intensity for recurrent glioblastoma-interim analysis and safety assessment (Optimalttf-1). Neuro-Oncology, 20(Suppl 6), vi21-vi21.

19. Wenger, C., Salvador, R., Basser, P. J., \& Miranda, P. C. (2015). The electric field distribution in the brain during TTFields therapy and its dependence on tissue dielectric properties and anatomy: A computational study. Physics in Medicine \& Biology, 60, 7339-7357.

20. Miranda, P. C., Mekonnen, A., Salvador, R., \& Basser, P. J. (2014). Predicting the electric field distribution in the brain for the treatment of glioblastoma. Physics in Medicine and Biology, 59 (15), 4137.

21. Saturnino, G. B., Antunes, A., \& Thielscher, A. (2015). On the importance of electrode parameters for shaping electric field patterns generated by tDCS. NeuroImage, 120, 25-35.

22. Korshoej, A. R., Hansen, F. L., Mikic, N., Thielscher, A., von Oettingen, G. B., \& JCH, S. (2017). Exth-04. Guiding principles for predicting the distribution of tumor treating fields in a human brain: A computer modeling study investigating the impact of tumor position, conductivity distribution and tissue homogeneity. Neuro-Oncology, 19(Suppl 6), vi73.

23. Korshoej, A. R., Hansen, F. L., Mikic, N., von Oettingen, G., JCH, S., \& Thielscher, A. (2018). Importance of electrode position for the distribution of tumor treating fields (TTFields) in a human brain. Identification of effective layouts through systematic analysis of array positions for multiple tumor locations. PLoS One, 13(8), e0201957.

24. Lok, E., San, P., Hua, V., Phung, M., \& Wong, E. T. (2017). Analysis of physical characteristics of Tumor Treating Fields for human glioblastoma. Cancer Medicine, 6, 1286.

25. Korshoej, A. R., Hansen, F. L., Thielscher, A., von Oettingen, G. B., \& Sørensen, J. C. H. (2017). Impact of tumor position, conductivity distribution and tissue homogeneity on the distribution of tumor treating fields in a human brain: A computer modeling study. PLoS One, 12(6), e0179214.

Open Access This chapter is licensed under the terms of the Creative Commons Attribution 4.0 International License (http://creativecommons.org/licenses/by/4.0/), which permits use, sharing, adaptation, distribution and reproduction in any medium or format, as long as you give appropriate credit to the original author(s) and the source, provide a link to the Creative Commons license and indicate if changes were made.

The images or other third party material in this chapter are included in the chapter's Creative Commons license, unless indicated otherwise in a credit line to the material. If material is not included in the chapter's Creative Commons license and your intended use is not permitted by statutory regulation or exceeds the permitted use, you will need to obtain permission directly from the copyright holder.

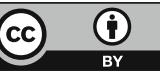

